\title{
Screening of PGPR from the Rhizosphere of Groundnut (Arachis hypogea): Characterization and Application
}

\author{
R. Nigar Sulthana*, A. Rajanikanth and M. Padamavathi \\ Department of Biotechnology, SPW Degree \& PG College, Tirupati, A.P, India \\ *Corresponding author
}

\section{A B S T R A C T}

\section{Keywords \\ Arachis hypogea, \\ Plant growth \\ promoting \\ rhizobacteria (PGPR), \\ PVK broth, Physico \\ chemical analysis, \\ Phosphate \\ solubilization \\ Article Info \\ Accepted: \\ 28 June 2018 \\ Available Online: \\ 10 July 2018}

Rhizosphere of a groundnut plant (Arachis hypogea) from agricultural fields of Chittoor district of Andhra Pradesh (India) was explored for isolation of PGPR. A total of 6 isolates from the rhizosphere belonging to different species were isolated. All the isolates showed phosphate solubilization, out of these an isolate coded as Psm6 showed most prominent halo zone was tested in vivo for growth promotion of Groundnut (Arachis hypogaea) under field conditions. Root and Shoot length, Dry and Wet weights were found to be excellent in Psm6 treatment compare to control. Psm6 treated plants showed increase in fresh biomass, dry biomass, root length and shoot length by $19 \%, 11 \%$, $10 \%$ and $12 \%$ respectively. Physicochemical properties of soil revealed that soil was slightly alkaline; Nitrogen and phosphorous contents were low in the soil. After treatment with PGPR they showed slight increase and excellent growth of Groundnut under field condition.

\section{Introduction}

The rhizosphere is the soil found around the root it is a site with complex interactions between the root and associated microorganisms. Rhizobacteria are the group of bacteria that colonize the rhizosphere naturally. These are soil bacteria that stimulate plant growth after inoculation of seeds or roots and beneficial interactions can be enhanced if these are effectively managed. The beneficial bacteria termed as plant growth promoting rhizobacteria (PGPR) stimulate growth of plants. The PGPR have been studied in various crop plants (Burr and Caesar 1983). Plant growth promoting rhizobacteria (PGPR) are functionally diverse group of bacteria having immense potential as biofertilizers and biopesticides. Depending upon their function, they may serve as partial replacements for chemical fertilizer or pesticides as an ecofriendly and cost-effective alternative as compared to their synthetic counterparts. Hence isolation, characterization and practical evaluation of PGPRs having multifaceted beneficial characteristics, are essentially required (Pradhan et al., 2017). Therefore, the objectives of this study were to isolate native 
bacterial strains from the groundnut (Arachis hypogaea) rhizosphere under in vitro conditions and to characterize these isolates for phosphate solubilization, to assess the PGPAs of these isolates in vivo and their eff ect on the nutrient contents ( $\mathrm{N}$ and $\mathrm{P}$ ) of groundnut plants at growth stage.

\section{Materials and Methods}

\section{Sample collection}

Soil samples were collected from rhizosphere of groundnut plants grown in fields of Chittoor district of Andhra Pradesh were uprooted carefully, shoot portion cut off and roots along with the rhizosphere soil aseptically in small plastic bags / bottles were brought to the laboratory and prior to their processing kept at $4^{\circ} \mathrm{C}$.

Screening and qualitative analysis of isolates for plant growth promoting activity

$10 \mathrm{~g}$ of soil samples was suspended in $90 \mathrm{ml}$ of sterilized distilled water and $10^{-}$

${ }^{1}$ dilution was obtained. Serial dilutions were prepared by mixing $1 \mathrm{ml}$ of the suspension made into $9 \mathrm{ml}$ sterilized water blanks until the $10^{-7}$ dilution was obtained.

From these dilutions $100 \mu 1$ was spread plated on Pikovskaya's Agar plates (Pikovskayas, 1948). These plates were then incubated at $30^{\circ} \mathrm{C}$ and were observed for 2-7 days. The total bacterial types were counted after 48 hours of incubation.

The Phosphate solubilizing bacteria (PSB) showing halo zones of clearance were streaked again on PVK agar plate to check for purity and Phosphate solubilizing ability. The pure strains forming zone of clearance were maintained by streaking on nutrient agar slants and stored at $4^{\circ} \mathrm{C}$.
Quantitative estimation of Phosphate solubilization in liquid medium

The phosphate in solution was determined by using Calorimetric Chlorostannous reduced molybdo phosphoric acid blue method (Jackson, 1973). The PSB were grown in $50 \mathrm{ml} \mathrm{NB}$ for 24 hours at $30^{\circ} \mathrm{C}$ in incubator shaker. $1 \mathrm{ml}$ of each PSB was aseptically transferred to $50 \mathrm{ml}$ of PVK broth contained in $150 \mathrm{ml}$ conical flask. The flasks were incubated at $30^{\circ} \mathrm{C}$ for $3-7$ days in incubator shaker at $120 \mathrm{rpm}$. After 3 days of incubation, $5 \mathrm{ml}$ culture was withdrawn from each flask and cultures were centrifuged at 10,000 rpm for 30min. The supernatant was diluted to 100 $\mathrm{ml}$ with autoclaved distilled water. Then $5 \mathrm{ml}$ aliquot of each dilution was transferred to $50 \mathrm{ml}$ volumetric flask. This was followed by addition of $10 \mathrm{ml}$ chloromolybdic acid, which was added along the sides of the flask. The contents of the flasks were diluted to $40 \mathrm{ml}$ with distilled water. Then 5 drops of chlorostannous acid was added. After mixing, the volume was made up to $50 \mathrm{ml}$ with distilled water. The blue colour intensity of the solution was measured in a spectrophotometer at O.D. $660 \mathrm{~nm}$. The soluble Phosphate was estimated from standard curve of $\mathrm{KH}_{2} \mathrm{PO}_{4}(0-2 \mathrm{ppm})$ drawn against O.D. $600 \mathrm{~nm}$.

\section{Field study}

The potential strain Psm6 showing good PGPR activity was tried with Groundnut for determination of effect on plant growth and crop productivity under field and natural environmental conditions. The isolate was grown in LB medium with agitation (125 rpm) for $48 \mathrm{~h}$ at $28^{\circ} \mathrm{C}$ to a final concentration of 108 $\mathrm{CFUml}^{-1}$.

Groundnut seeds were then inoculated with bacterial suspension for $30 \mathrm{~min}$ at room temperature. Control seeds were treated in the same manner with uninoculated LB medium. 
The parameters evaluated were dry weight, wet weight, root length and shoot length (Rocheli de Souza et al., 2012).

\section{Physiochemical analysis of soil samples}

To validate the potency of the bacterial PGPR activity field experiment was conducted where various analysis were done to evaluate strength of the soil before and after field experiment. Analysis of physico-chemical properties such as $\mathrm{pH}$, Electrical conductivity (EC), available phosphorus, organic carbon and organic matter (OC/OM), available nitrogen of Groundnut field soil was analysed by standard method (DIRD, Pune, 2009).

\section{Results and Discussion}

Plant growth promoting activity of the bacterial isolates

In search of efficient plant growth promoting activity, a total of 6 bacterial strains were isolated and checked their activity on phosphate solubilization. All the strains were positive to phosphate solubilization and showed halo zones on PVK agar plates (fig.1).

Screening of bacterial isolates for phosphate solubilization revealed variations among different groups of organisms. Fig. 2 shows the selection of efficient Phosphate Solubilizing Bacterial isolates on qualitative basis. Similar criteria of selection of efficient PSB were followed by Oswal and Bhide, (1972).

\section{Phosphate Solubilization in liquid medium}

After confirming the Phosphate Solubilizing Activity on solid medium, the phosphorus solubilization was confirmed quantitatively in liquid medium using (PVK Broth). Different investigators have used various media for studying phosphate solubilization in liquid medium. Pradhan and Sukla, (2006) found a suitable medium formulation as an ideal one for new isolates. Considering amount of glucose used in medium and corresponding efficacy of Phosphate solubilization, PVK medium proved to be most effective without compromising the solubilization.

All bacteria tested were found to be solubilizers of Tri calcium phosphate in PVK broth. The Phosphate content released into the medium from Tri calcium phosphate were given in the Fig.3. Results revealed that an isolate coded as Psm-6 showed maximum Phosphate solubilization in liquid medium. It was evident that in the medium with Tri calcium phosphate, the values of dissolved phosphate obtained with the isolate was convincingly showing that the tested isolate have effectively converted the inorganic, insoluble phosphate into soluble form and was selected for further studies.

Incubation in PVK broth supplemented with tri calcium phosphate, Phosphorus solubilizing bacteria are reported to dissolve insoluble phosphates by production of inorganic or organic acids and/or by the decrease of the $\mathrm{pH}$ (Whitelaw, 2000). Most of the previous reports stated that calcium phosphates are dissolved by acidification. Therefore any microorganism that acidifies its external medium will show some level of Phosphate Solubilizing Activity (Pradhan and Sukla, 2006). It is well known that Phosphate Solubilizing Bacteria in soil solubilize insoluble phosphates mainly by secreting acids into the medium (Dave and Patel, 2003). Isolates showed maximum Phosphate solubilization activity might have used the same mechanism to solubilize the insoluble form of phosphate into soluble form. Similar method was also used by Achal et al., (2007) to analyze the soluble content of phosphate in 
culture filtrate of Aspergillus tubingensis analyze the soluble content of phosphate in and by Himani and Reddy, (2011) to culture supernatant of Bacillus sp.

Table.1 Physico chemical properties of soil before and after treatment of PGPR in Groundnut field

\begin{tabular}{|l|c|c|}
\hline Physico chemical properties & \multicolumn{2}{|c|}{ Isolate code } \\
\cline { 2 - 3 } & Control & Psm6 \\
\hline pH & 8.1 & 8.3 \\
\hline Electrical conductivity $(\boldsymbol{\mu} \mathbf{~ s / c m )}$ & 0.17 & 0.25 \\
\hline Organic matter $(\mathbf{k g} / \mathbf{g}$ of soil) & 5.9 & 6.2 \\
\hline Total nitrogen $(\mathbf{k g} / \mathbf{h})$ & 1.15 & 1.19 \\
\hline Phosphorus $(\mathbf{k g} / \mathbf{h})$ & 119 & 123 \\
\hline Carbon $(\mathbf{k g} / \mathbf{h})$ & 9.5 & 9.8 \\
\hline
\end{tabular}

Fig.1 Screening of Bacteria for Phosphate solubilization on Pikovskayas Agar Medium

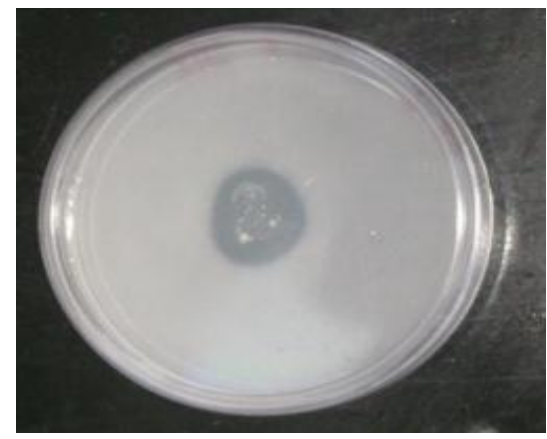

Fig.2 Phosphate solubilization by Bacterial isolates

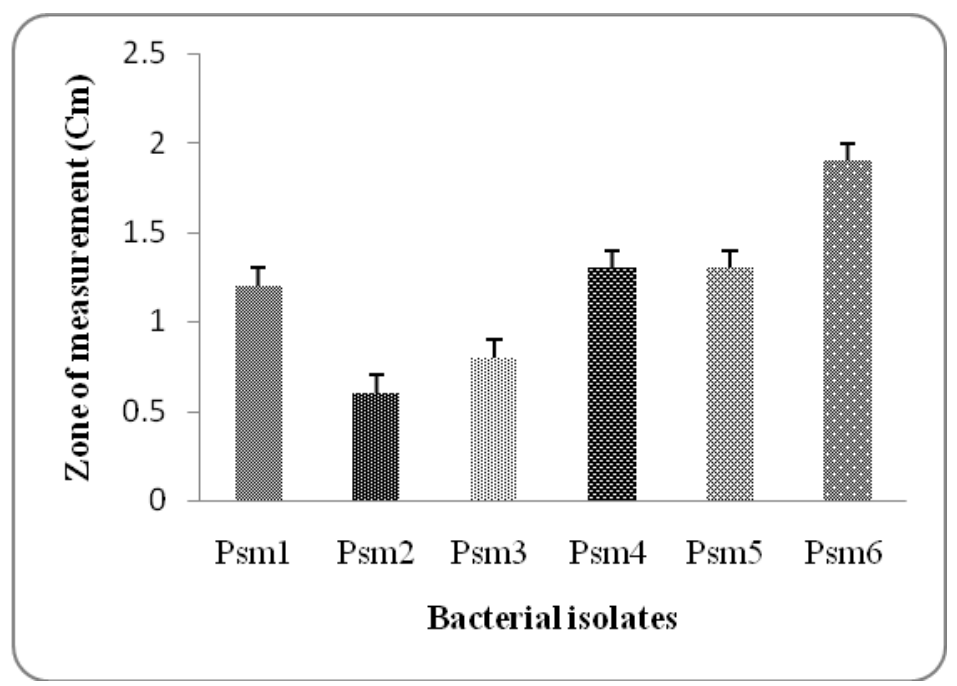


Fig.3 Phosphate solubilizing activity of Bacterial isolates

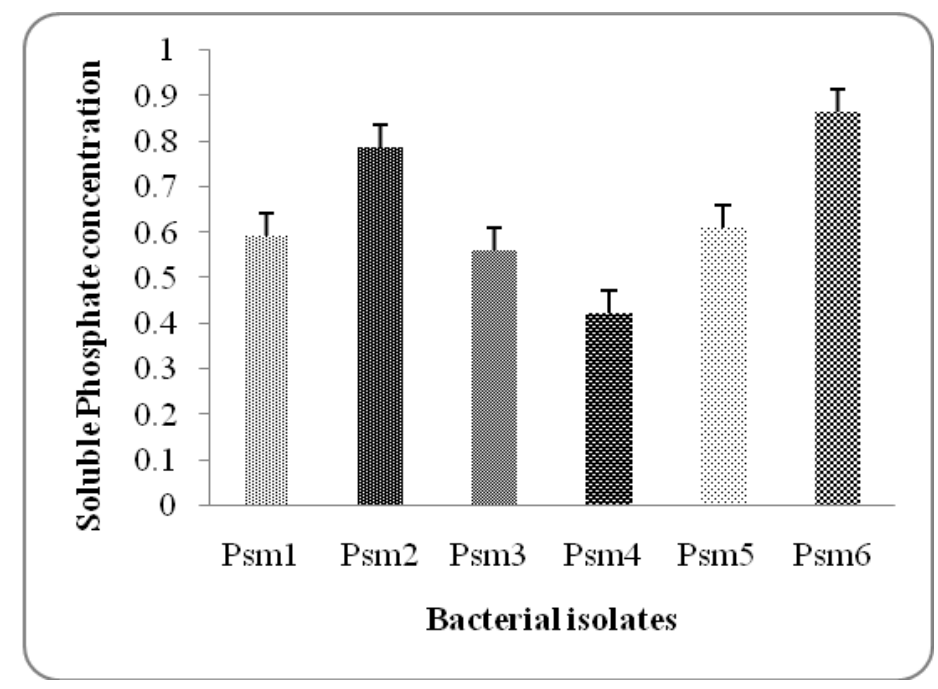

Fig.4 Growth of control and PGPR treated ground nut in field condition

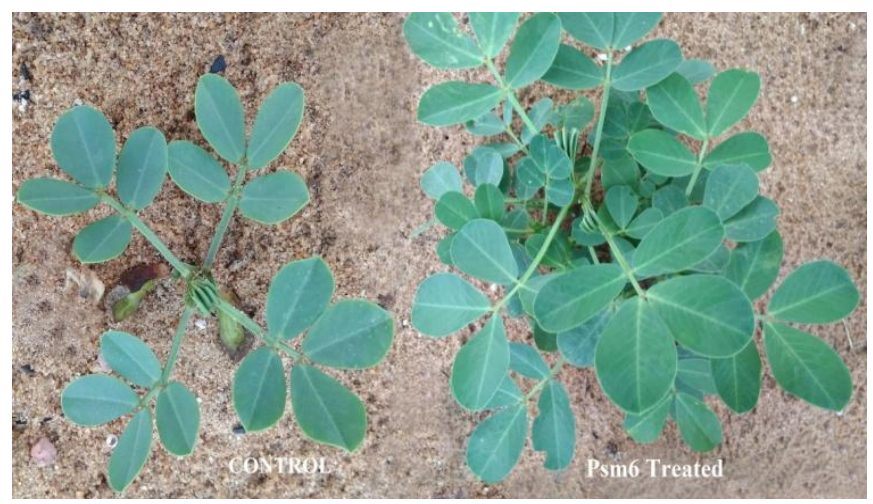

Fig.5 Dry weight, wet weight, root length and shoot length of control and PGPR treated Groundnut in field conditions

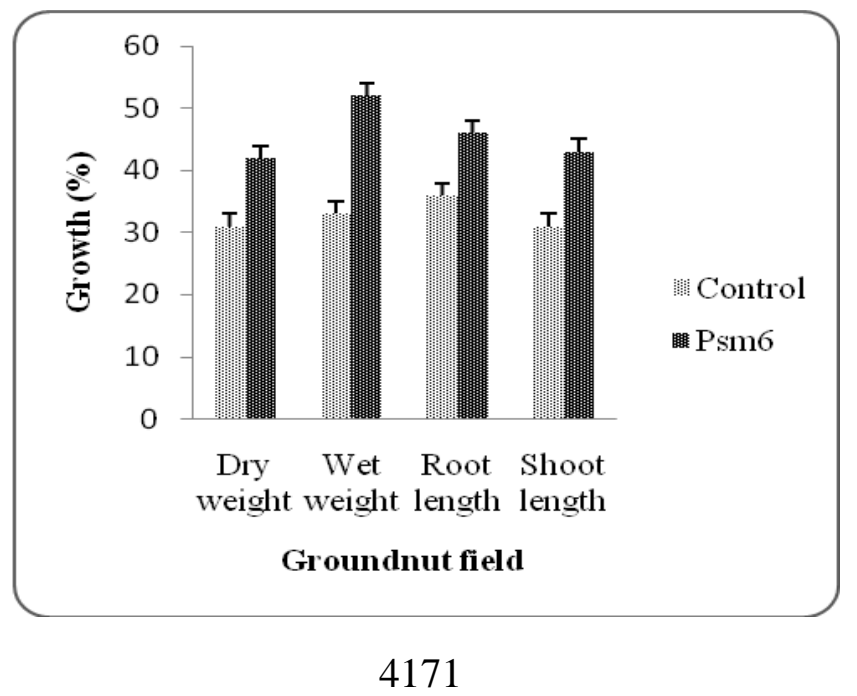




\section{Field study}

Biometric observation of Groundnut increases with treatment of Psm6 compare to control. Root and Shoot length, Dry and Wet weights were found to be excellent in Psm6 treatment compare to control (fig.4). Field study results revealed that there was significant increase in plant growth, Root length, Shoot length, Dry weight, Wet weight were of Groundnut with the inoculation of selected PGPR strains. When compared with PGPR treated groundnut, the control plant grown in field conditions showed that dry weight of the plant was reduced by $11 \%$ and fresh biomass was reduced by $19 \%$ and root length and shoot length of $10 \%$ and $12 \%$ (fig.5). This results of present study clearly showed the efficiency of Psm6 in plant growth enhancement, phosphorus uptake and soil fertility. Many studies in relation to crop improvement by PGPR were carried out either in pot cultures or field conditions [3, $16]$.

\section{Physiochemical properties of soil}

Physico chemical properties of soil used for field study was determined. After Psm6 inoculums treatment the physical-chemical properties such as $\mathrm{pH}$, Electrical Conductivity, Organic Carbon and organic matter, Nitrogen and available Phosphorus were also increased significantly from control in Groundnut at field conditions (Table.1). From several studies and literature review it has been found that after treatment of PGPR as bio inoculants organic carbon, nitrogen and phosphorus also increases in the soil environment (Gunasekaran et al., 2004) with slight reduction in $\mathrm{pH}$ (Shinde et al., 2008). It has already been reviewed that production of organic acids by soil microorganisms and commensurate $\mathrm{pH}$ decrease is the major mechanism of phosphate solubilization (Whitelaw MA, 2000).
In conclusion, soil was slightly alkaline and belongs to low salinity class and are neutral in nature. Nitrogen and phosphorous content was low in the soil; organic matter was medium in the sample. After addition of PGPR isolate not only show in-vitro activity but they are showing excellent growth of plants in natural environmental condition. Among all isolates Psm6 exhibited better activities, which can be directed for farming. However, a lot of research is needed for evaluating the biotechnological properties of these bacterial species showing PGPR activity.

\section{Acknowledgement}

The Authors are great full to, Department of Biotechnology, SPW Degree and PG College for providing funding and field for the experiment.

\section{References}

Achal, V. Savant, V.V. and Sudhakara Reddy, M. 2007. Phosphate Solubilization by Wide Type Strain and UV-induced Mutants of Aspergillus tubingensis. Soil Biology and Biochemistry, 39(2): 695-699.

Burr, TJ. and Caesar, A. 1983. Beneficial plant bacteria. Critical Reviews in Plant Science, 2:1-20.

Dave, A. and Patel, HH. 2003, Impact of different carbon and nitrogen sources on phosphate solubilization by Pseudomonas fluorescens. Indian Journal of Microbiology. 43: 33-36.

Directorate of Irrigation Research \& Development, Pune. 2009. Laboratory Testing Procedure for Soil and Water Sample Analysis, ISO 9001:2000 Certified.

Gunasekaran, S. Balachandar, D. Sundaram, KM. 2004. Studies on synergism between Rhizobium, plant growth 
promoting rhizobacteria (PGPR) and phosphate solubilizing bacteria in Blackgram. Biofertilizers technology, 269-72.

Himani, S. Reddy, MS. 2011. Effect of inoculation with phosphate solubilizing fungus on growth and nutrient uptake of wheat and maize plants fertilized with rock phosphate in alkaline soils. European Journal of Soil Biology. 47: 30-34.

Jackson, ML. 1973, Methods of chemical analysis. Prentice Hall of India (Pvt.) Ltd., New Delhi.

Ostwal, KP. and Bhide, VP. 1972. Solubilization of tricalcicum phosphate by soil Pseudomonas. Indian Journal of Experimental Biology, 10:153-4.

Pikovskaya, RI. 1948. Mobilization of phosphorous in soil connection with the vital activity of some microbial species. Microbiologiya, 17: 362-370.

Pradhan, A., Mohapatra, S., Mohanty, D., Samantaray, D. and Mishra, BB. 2017. Effect of polyhydroxyalkanoates accumulated plant growth promoting Bacillus sp. on germination and growth of Mung Bean and Groundnut. Research Journal of Pharmaceutical,
Biological and Chemical Sciences, 8(4): 789-797.

Pradhan, N. and Sukla, LB. 2006. Solubilization of inorganic phosphate by fungi isolated from agriculture soil. African Journal of Biotechnology, 5: 850-854.

Rocheli de Souza, Anelise Beneduzi, Adriana Ambrosini, Pedro Beschoren da Costa, Jacqueline Meyer, Luciano K. Vargas, Rodrigo Schoenfeld, Luciane M. P. Passaglia. 2012. The effect of plant growth-promoting rhizobacteria on the growth of rice (Oryza sativa L.) cropped in southern Brazilian fields. Plant Soil, DOI 10.1007/s11104-0121430-1.

Shinde, DB. Vahini, C. Jadhav,AC. 2008. Influence of plant growth promoting rhizobacteria (PGPRs) on nutrient availability and rhizobacterial population in groundnut cropped soil. Journal of Maharashtra agricultural universities, 33: 335-38.

Whitelaw, MA. 2000. Growth promotion of plants inoculated with phosphate solubilizing fungi. Edited by Donald L. Sparks. Advances in Agronomy, Academic press, 69: 99- 151.

\section{How to cite this article:}

Nigar Sulthana, R., A. Rajanikanth and Padamavathi, M. 2018. Screening of PGPR from the Rhizosphere of Groundnut (Arachis hypogea): Characterization and Application. Int.J.Curr.Microbiol.App.Sci. 7(07): 4167-4173. doi: https://doi.org/10.20546/ijcmas.2018.707.486 